uveitis involving the right eye for the last 2 years. His first evaluation 2 weeks into the illness showed reduced visual acuity (20/200) in the right eye, the left eye being normal $(20 / 20)$. The anterior chamber showed flare with hazy media. Evaluation for sarcoid, tuberculosis, syphilis and vasculitis was negative. He was initiated on steroids, with total improvement over 4 weeks. He later discontinued steroids due to weight gain. One month after stopping steroids, he developed redness of the same eye with visual deterioration. Evaluation showed exudative retinal detachment. Eventually, he developed complicated cataract and prepthisical changes. He also had two episodes of low-grade fever with headache and terminal neck stiffness without altered sensorium or focal deficits and was treated symptomatically. Examination at the present admission revealed patches of vitiligo on his back. Visual acuity was 20/20 in the left eye and no perception of light in the right eye. Ophthalmic evaluation showed phthisis bulbi and complicated cataract in the right eye [Figure1]. The left eye showed optic disc edema, tortuous veins and choroidal folds [Figure 2]. Neurological examination showed gaze-

\title{
Intracerebral hemorrhages in Vogt-Koyanagi-Harada disease
}

Sir,

Vogt-Koyanagi-Harada (VKH) disease is a multisystem disorder characterized by granulomatous panuveitis with exudative retinal detachments often associated with neurologic, acoustic and cutaneous manifestations.$^{[1]}$ The neurological features of VKH disease include headache, meningismus, cranial nerve palsies, hemiparesis, transverse myelitis and ciliary ganglionitis. ${ }^{[1]}$ Intracerebral hemorrhage has not been described earlier. We describe a male patient with VKH disease who presented with ataxia secondary to intracerebral hemorrhage.

A 26-year-old gentleman presented with acute onset headache, vomiting, imbalance while walking and incoordination of the upper limbs. There was no history of weakness, sensory impairment or any cranial nerve deficit. He gave a history of recurrent nontraumatic

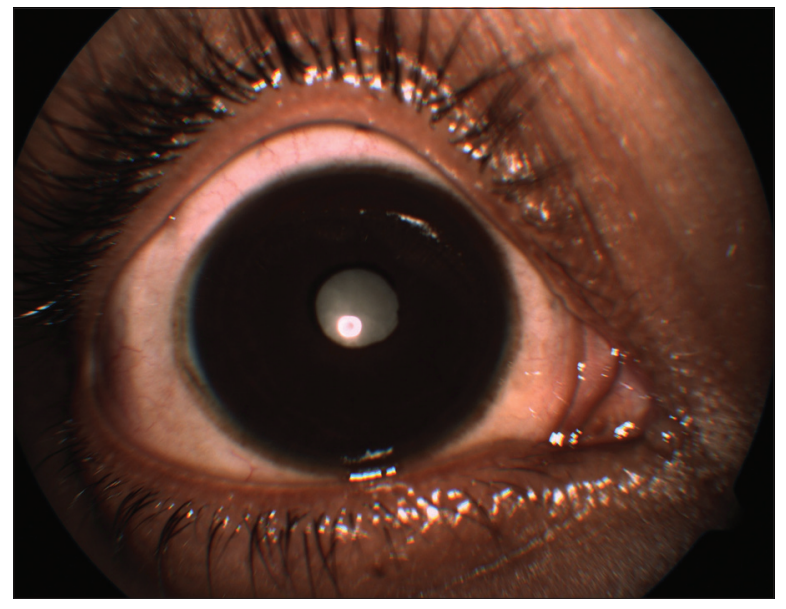

Figure 1: Complicated cataract in the right eye

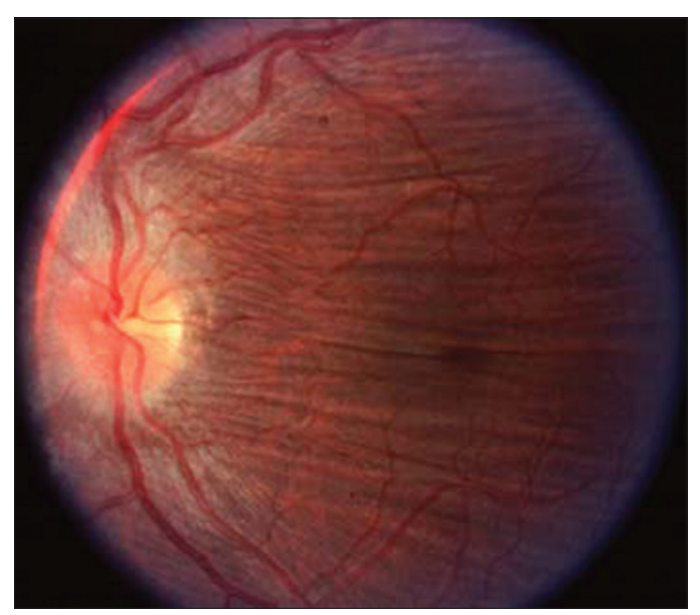

Figure 2: Fundus picture of the left eye showing optic disc edema with choroidal folds and tortuous veins 


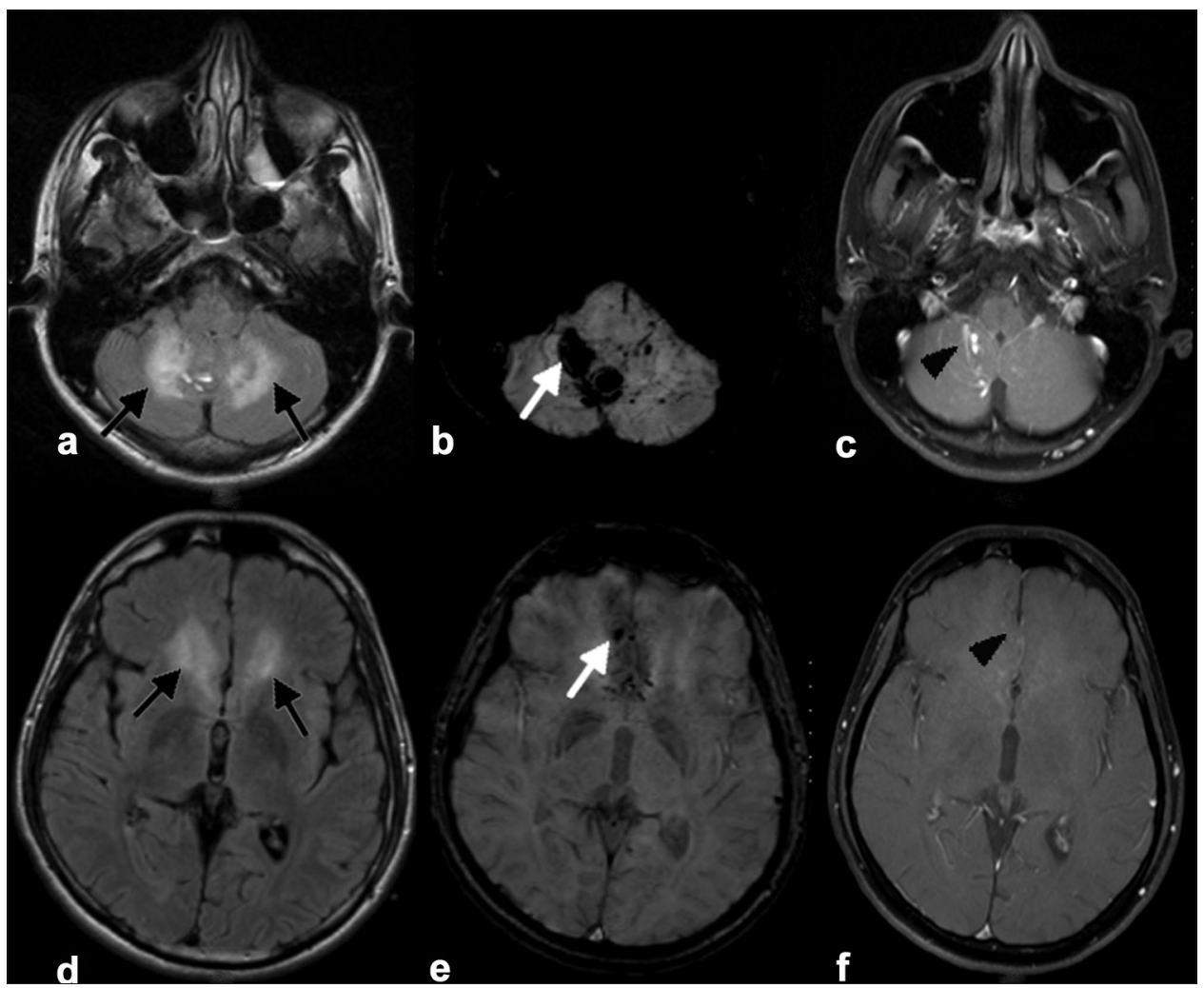

Figure 3: 1.5 Tesla magnetic resonance imaging of the brain. Axial flair images (a-d) show hyperintensities involving bilateral cerebellar hemispheres, vermis, basifrontal region and genu of corpus callosum (black arrows). SWI axial images (b-e) show susceptibility effects in the right cerebellar hemispheres, vermis and basifrontal region (white arrows). Postcontrast T1-weighted axial images (c-f) show leptomeningeal enhancement of the right cerebellar and basifrontal region (black arrowheads)

evoked nystagmus and bilateral upper and lower limb incoordination with gait ataxia.

Cerebrospinal fluid (CSF) showed pleocytosis 90 cells $/ \mathrm{mm}^{3}$ (100\% lymphocytes) with normal sugar (CSF: Plasma glucose 0.65) and mildly elevated protein $(64 \mathrm{mg} / \mathrm{dl})$. Audiometry showed bilateral mild sensorineural hearing loss. Ultrasonography B-mode scan of the right eye showed cone-shaped retinal detachment with subretinal hemorrhage. Biopsy from the eye was withheld because of risk of sympathetic ophthalmitis. Magnetic resonance imaging (MRI) of the brain [Figure 3] showed bilateral cerebellar, vermian and basifrontal bleed with mild leptomeningeal enhancement on postcontrast T1weighted images. Four-vessel digital subtraction angiogram was normal. He responded excellently to a course of intravenous steroids and was continued on oral steroids. On discharge, he could walk normally and was asymptomatic at the 3-month follow-up.

$\mathrm{VKH}$ syndrome is a rare systemic disease involving melanocyte-containing organs. VKH occurs more commonly in patients with a genetic predisposition to the disease, including Asian, Middle Eastern, Hispanic and Native American populations. ${ }^{[1]}$ The highest prevalence is in Japan, where VKH disease represents $6.8-9.2 \%$ of all patients with uveitis. ${ }^{[1]} \mathrm{VKH}$ is related to an aberrant $\mathrm{T}$ cell-mediated immune response directed against selfantigens found on melanocytes. Yamaki and coworkers have shown that the tyrosinase-related proteins, TRP1 and TRP2, can induce disease in Lewis rats that is similar to VKH disease in humans. ${ }^{[2]}$ Thus, any organ containing melanocytes or tyrosinase can be affected in $\mathrm{VKH}$ disease. The International Committee on Nomenclature has established revised criteria for the diagnosis of $\mathrm{VKH}$ disease. ${ }^{[3]}$ Our patient satisfied the complete diagnostic criteria for VKH. A literature search reveals infrequent case reports of neurological complications. An early case report of VKH with vestibular and cerebellar ataxia and another with multiple cranial nerve palsies has been reported, which improved with steroid therapy, but imaging was not performed. ${ }^{[4]}$ Osaki et al. have reported the case of a 57-year-old Japanese male with VKH who presented with truncal ataxia in whom MRI revealed cerebellar vermian contrast enhancement. ${ }^{[5]}$

Our patient had multiple areas of parenchymal bleed confirmed by SWI and leptomeningeal enhancement on postcontrast MRI. Intracerebral bleed in our case might have resulted from T cell-mediated cytotoxicity along with humoral autoimmune response directed against tyrosinase-related proteins found in the neurons. Immune-mediated damage breaks the blood 
brain barrier, which manifested as leptomeningeal enhancement. The fact that he made excellent recovery to immunomodulatory therapy highlights the need for prompt recognition and management to avoid morbidity.

Neeraj N. Baheti, Ajith Cherian, Mahesh Kate, Syam Krishnan, Bejoy Thomas ${ }^{1}$

Departments of Neurology and ${ }^{1}$ Imaging Sciences and Interventional Radiology, Sree Chitra Tirunal Institute for Medical Sciences and Technology, Trivandrum, Kerala, India. E-mail: drajithcherian@yahoo.com

\section{References}

DOI: $10.4103 / 0028-3886.59491$

1. Moorthy RS, Inomata H, Rao NA. Vogt-Koyanagi- Harada syndrome. Surv Ophthalmol 1995;39:265-92.

2. Yamaki K, Gocho K, Hayakawa K, Kondo I, Sakuragi S. Tyrosinase family proteins are antigens specific to Vogt-Koyanagi-Harada disease. J Immunol 2000;165:7323-9.

3. Read RW, Holland GN, Rao NA, Tabbara KF, Ohno S, ArellanesGarcia L, et al. Revised diagnostic eriteria for Vogt-Koyanagi-Harada disease: Report of an international committee on nomenclature. Am J Ophthalmol 2001;131:647-52.

4. Hiraki Y, Kuwasaki N, Shoji H, Kaji M, Kuboshiro T. Each one case of Vogt-Koyanagi-Harada disease with vestibular and cerebellar ataxia, and multiple cranial nerve palsies. Rinsho Shinkeigaku 1989;29:54-8.

5. Osaki Y, Matsubayashi K, Doi Y, Ozawa T. Vogt-Koyanagi-Harada disease with cerebellar lesions demonstrated on MRI: A case report. Rinsho Shinkeigaku 1995;35:1160-2.

Accepted on 12-10-2009 